\title{
Second-hand smoke exposure in a sample of European hospitals
}

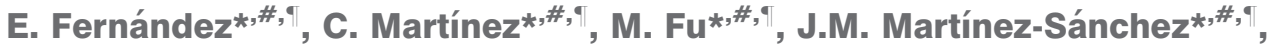 \\ M.J. López ${ }^{+, \S}$, G. Invernizzi ${ }^{\dagger}$, A. Ouranou**, B. Dautzenberg** and M. Nebot ${ }^{+, \S, \# \# ~}$
}

ABSTRACT: Smoking in hospitals is banned in many European countries; nevertheless, the level of compliance is diverse, and, in some cases, smoking areas remain. The present study describes the levels of second-hand smoke, as derived from respirable suspended particle measurements, in a sample of European hospitals during the year 2007.

The present study was a multicentric descriptive cross-sectional study carried out in 30 hospitals in seven European countries (Austria, Belgium, France, Germany, Greece, Romania and Spain). Particulate matter with a $50 \%$ cut-off aerodynamic diameter of $2.5 \mu \mathrm{m}$ (PM2.5) concentration was measured by means of a hand-held laser-operated monitor of particle size and mass concentration in six selected indoor locations. Medians and interquartile ranges of PM2.5 concentration were computed in order to describe the data by country and location of measurement.

The median PM2.5 concentration in all countries and locations was $3.0 \mu \mathrm{g} \cdot \mathrm{m}^{-3}$, with half of the measurements ranging $2.0-7.0 \mu \mathrm{g} \cdot \mathrm{m}^{-3}$. PM2.5 levels were similar across countries. Eleven $(5.5 \%)$ measurements were $>25.0 \mu \mathrm{g} \cdot \mathrm{m}^{-3}$, which is the 24-h mean limit recommended by the World Health Organization outdoor air quality guideline.

The present results show that exposure to second-hand smoke in this sample of European hospitals is very low, and can be easily monitored in order to ensure smoke-free legislation compliance.

KEYWORDS: Environmental tobacco smoke, Europe, hospitals, particles with a $50 \%$ cut-off aerodynamic diameter of $2.5 \mu \mathrm{m}$, second-hand smoke, tobacco smoke pollution

econd-hand smoke (SHS) or exposure to environmental tobacco smoke has important public health implications. It has been classified as a lung carcinogen [1], and has been proven to have adverse health effects on children and adults, including heart disease, lung cancer and other respiratory disorders [2].

Smoking in hospitals is completely banned in many European countries by national or regional laws [3]. In these countries, as well as in countries without complete bans on smoking, some hospitals have opted to go smoke-free on their own initiative or in association with national networks integrated within the European Network of Smoke-free Hospitals (ENSH). The ENSH is a nongovernmental organisation coordinating national and regional smoke-free networks in 20 European countries including $\sim 1,400$ hospitals. The ENSH promotes common strategies for obtaining tobacco-free environments and providing active support for quitting by patients, visitors and staff among European hospitals. ENSH activities are based on a European code of smoke-free hospitals and health services, providing various tools to support successful implementation of tobacco-free policies in health facilities [4].

To date, few studies have used direct measurements of SHS to monitor the accomplishment of the smoke-free hospital policy [5, 6]. Exposure to SHS has been measured by various methods, such as questionnaires (based on self-reports) and markers of SHS, namely substances found in tobacco smoke (such as nicotine) that can be measured in body fluids (urine, blood and saliva) or in the air to provide an objective measure of SHS exposure [7]. Airborne markers, such as vapour-phase nicotine or respirable suspended particles indicate the mean exposure level in a specific setting, and are easier to obtain than biological samples [8]. Among respirable suspended particles, those with a 50\% cut-off aerodynamic diameter of $2.5 \mu \mathrm{m}$ (commonly known as fine particles or PM2.5) are widely used for SHS assessment in enclosed settings [9-11]. PM2.5 originate from all types of combustion,

\section{AFFILIATIONS}

*Tobacco Control Research

Programme, Institut Català

d’Oncologia-Institut d'Investigació

Biomèdica de Bellvitge, L'Hospitalet de Llobregat,

\#Dept of Clinical Sciences, Campus de Bellvitge, Universitat de Barcelona, L'Hospitalet de Llobregat, "Catalan Network of Smoke-free Hospitals

${ }^{+}$Evaluation \& Intervention Methods Unit, Agència de Salut Pública de Barcelona,

\#\# Dept of Experimental and Health Sciences, Universitat Pompeu Fabra Barcelona, and

${ }^{\S}$ CIBER de Epidemiología y Salud Pública, Spain.

${ }^{f}$ Tobacco Research Unit, Istituto Nazionale dei Tumori/ltalian College of General Practitioners, Milan, Italy. **European Network of Smoke-free Hospitals, Paris, France.

CORRESPONDENCE

E. Fernández

Tobacco Control Research Unit Institut Català d'Oncologia Av. Gran Via de l'Hospitalet 199-203

08907 L'Hospitalet de Llobregat Barcelona

Spain

E-mail: efernandez@ico.scs.es

Received

Nov 282008

Accepted after revision: Feb 102009

First published online: Feb 272009 
including motor vehicles, residential wood burning, forest fires, some industrial processes, etc. Although PM2.5 may derive from particles of dust and other combustion activities, smoking is generally the greatest contributor to indoor air pollution [12]. The aim of the present study was to describe the levels of SHS, as derived from PM2.5 measurements, in a sample of European hospitals during the year 2007.

\section{MATERIAL AND METHODS}

The present study was a multicentric descriptive crosssectional study among a convenience sample of 30 hospitals in seven European countries with different smoking prevalence rates and tobacco control activity (table 1). One hospital from Austria, five from Belgium, three from France, five from Germany, seven from Greece, four from Romania and five from Spain were included. Most of the hospitals were in urban areas and were general and specialised (maternity, oncological and children's) hospitals. Most of them were affiliated to a university (nursing or medical school), and all were members of the ENSH. The national coordinator of the smoke-free network in each country asked various hospitals to participate, taking into account the limited time-frame for making the measurements in each country (because the particle monitor had to go from one country to another; see below). The initial goal was to include five hospitals in as many countries as possible, and collaboration from seven countries was finally obtained.

A common protocol (derived from a previous study [6]) was used to sample and record the PM2.5 measurements. Six standard locations were defined within each hospital for measurement performance by centrally trained investigators: main entrance hall, emergency department waiting room, internal or general medicine hospitalisation unit, general surgery hospitalisation unit, cafeterias, and fire escapes. In addition, measurements were taken in other areas using the local investigator's criteria when the standard sampling areas were not available. Smoking areas in hospitals with these zones were also measured. Except in halls, all locations were unaffected by air flows that can potentially influence the distribution of particles in the air. For each PM2.5 measurement, the following data were recorded: hospital and location, date of measurement, sampling area, sampling volume, ventilation, and signs of smoking (tobacco smell, cigarette butts on the floor, and presence of ashtrays and persons smoking). Since the study only involved environmental measurements and not interventions or measurements in humans, approval from ethics committees was not required.

The PM2.5 concentration was measured with a pre-calibrated hand-held laser-operated monitor of particle size and mass concentration (Aerocet 531; Metone Instruments, Inc., Grants Pass, OR, USA) [14]. The operation was manual, with a userfriendly interface. The device was used with a short length of Tygon on a flat surface, not on the floor of the room, preferably in the middle, and away from any doors or windows. Owing to logistic constraints and because all locations were indoors, short (2 $\mathrm{min})$, for a mass-sample type, monitoring sessions were carried out in each location. The device displayed PM2.5 concentration and relative humidity on its screen, which were recorded by the same device and then transferred to a computer in the coordinating centre. The hospitals were sampled between March and July 2007 in all of the countries except for Romania (September to October 2007). The measurements were performed over 1-2 weeks consecutively by the local researcher, using the same device in the seven countries.

Given the skewed distribution of the PM2.5 concentrations, medians and interquartile ranges were computed to describe the data, and boxplots with logarithmic scales used to graphically present the distribution of PM2.5 concentrations by country and location. Tests for linearity were performed in order to explore the trends in PM2.5 concentrations by signs of smoking.

\section{RESULTS}

A total of 199 PM2.5 measurements were obtained within 30 hospitals across seven European countries: 30 in halls or main hospital entrances, 29 in emergency department waiting rooms, 22 in internal or general medicine hospitalisation units, 27 in cafeterias, 22 in fire escapes, 22 in general surgery hospitalisation units, and 39 in other places, including eight smoking areas (Belgium and Greece).

The overall median PM2.5 concentration was $3.0 \mu \mathrm{g} \cdot \mathrm{m}^{-3}$, with half of the measurements ranging $2.0-7.0 \mu \mathrm{g} \cdot \mathrm{m}^{-3}$. Similar PM2.5 levels were found across countries (table 2), with the lowest median concentration occurring in Germany (five hospitals

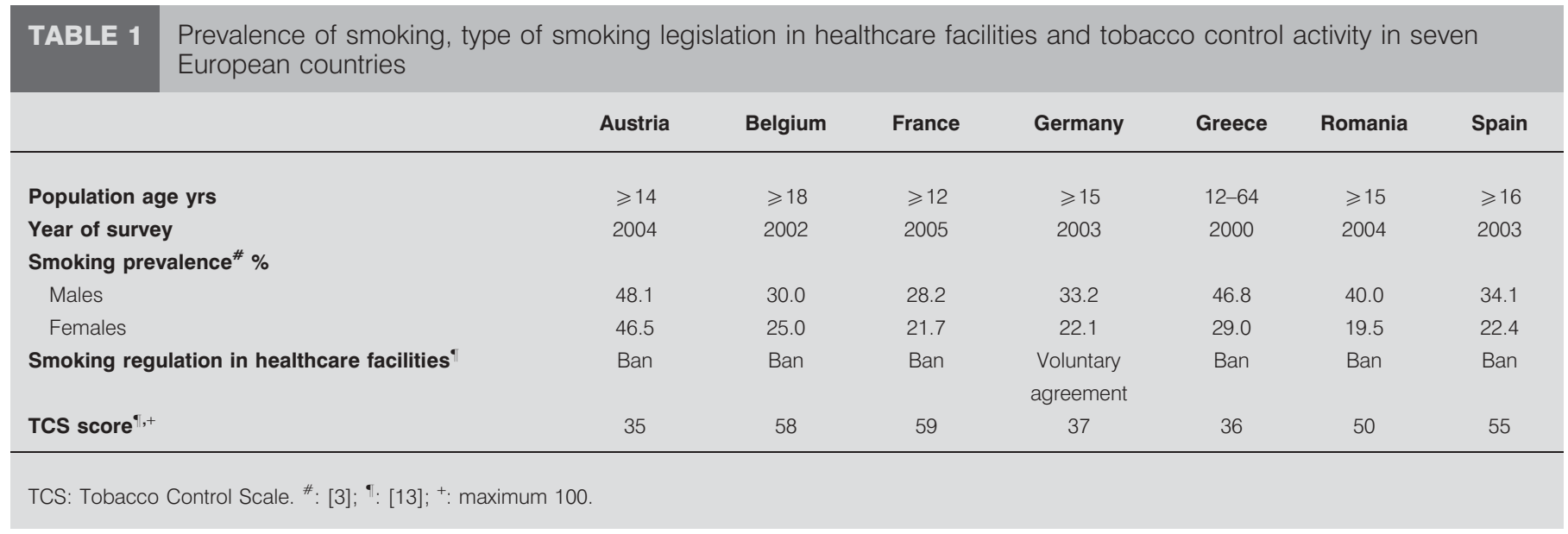


with a total of 30 measurements) and the highest in Romania (four hospitals with a total of 24 measurements). Eleven measurements were above the accepted 24-h mean limit recommended by the World Health Organization (WHO) outdoor air quality guideline $\left(25.0 \mu \mathrm{g} \cdot \mathrm{m}^{-3}\right)$ (fig. 1) [15], and five measurements were above the level recommended by the US Environmental Protection Agency $\left(35.0 \mu \mathrm{g} \cdot \mathrm{m}^{-3}\right)$ [16]. These measurements were taken in cafeterias, smoking areas and other zones in hospitals in Greece, Belgium and Romania, respectively.

The median PM2.5 concentrations in all of the countries by location ranged between 2.0 (surgery hospitalisation units) and $4.0 \mu \mathrm{g} \cdot \mathrm{m}^{-3}$ (internal medicine hospitalisation units) (table 2). Half of the measurements provided concentrations ranging $2.0-8.0 \mu \mathrm{g} \cdot \mathrm{m}^{-3}$, with a few levels of $>10.0 \mu \mathrm{g} \cdot \mathrm{m}^{-3}$ in halls, waiting rooms in emergency departments, internal medicine hospitalisation units, cafeterias and fire escapes (fig. 2). There were no wide variations across the seven countries, with the exception of Greece, Spain and Romania, which presented relatively higher concentrations. The measurements taken in smoking areas showed the highest median PM2.5 levels (i.e. $55.5 \mu \mathrm{g} \cdot \mathrm{m}^{-3}$ in Belgium), with some levels of $>60 \mu \mathrm{g} \cdot \mathrm{m}^{-3}$. The median PM2.5 concentration in locations with no signs of smoking was $4.0 \mu \mathrm{g} \cdot \mathrm{m}^{-3}$ (interquartile range: $2.0-8.0 \mu \mathrm{g} \cdot \mathrm{m}^{-3}$ ), and significantly increased to $6.0 \mu \mathrm{g} \cdot \mathrm{m}^{-3}$ (interquartile range $4.0-32.5 \mu \mathrm{g} \cdot \mathrm{m}^{-3}$ ) when all smoking signs were present $(p=0.020$ (test for linearity)).

\section{DISCUSSION}

The present study shows, for the first time with a European perspective, that levels of exposure to SHS in hospitals, as measured by PM2.5 concentration, are relatively low and without striking differences across countries. Most of the countries in the present study had passed specific smoking bans for healthcare facilities at the time of the study [3]. Some of these bans, however, had exceptions and permitted smoking in designated rooms within hospitals or even cafeterias (with or without smoking areas). Those locations with concentrations of $>25 \mu \mathrm{g} \cdot \mathrm{m}^{-3}$ were smoking zones, one cafeteria located in a separate building next to the hospital and other zones. These other zones included areas with restrictions on smoking (such as consultation rooms, patient rooms and doctors' offices), and hence indicates infringement of the smoke-free policy. Although PM2.5 detected in cafeterias might also originate from cooking in kitchens, most of the cafeterias did not have cooking facilities, and all of them had well-functioning built-in ventilation systems.

There are several particulate matter health effects on the respiratory and cardiovascular systems in children, adults and susceptible groups within the general population, and the epidemiological evidence shows adverse effects of particles after both short- and long-term exposure [17]. The present results show low overall PM2.5 levels in hospital facilities; nevertheless, the risk of various outcomes increases with exposure, and there is little evidence suggesting a threshold below which no adverse health effects would be anticipated [17]. Thus, according to the WHO air quality guideline, the aim must be to achieve the lowest concentrations possible in order to minimise risk effects.

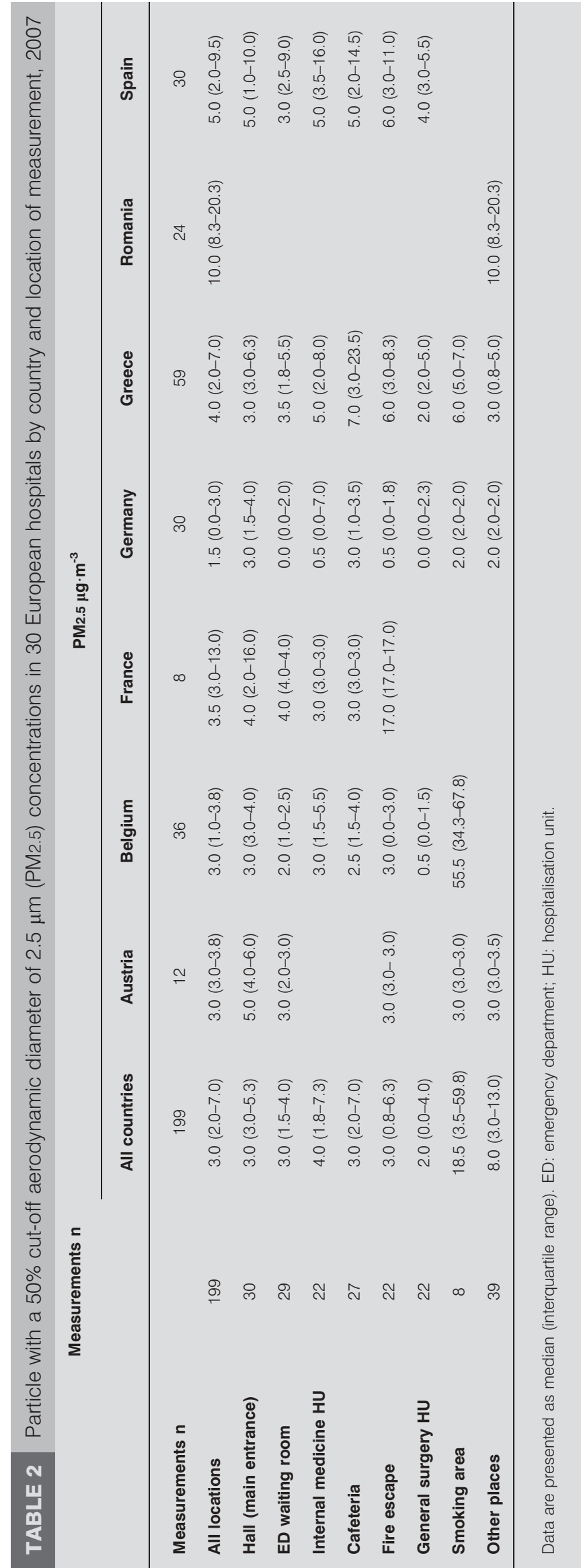




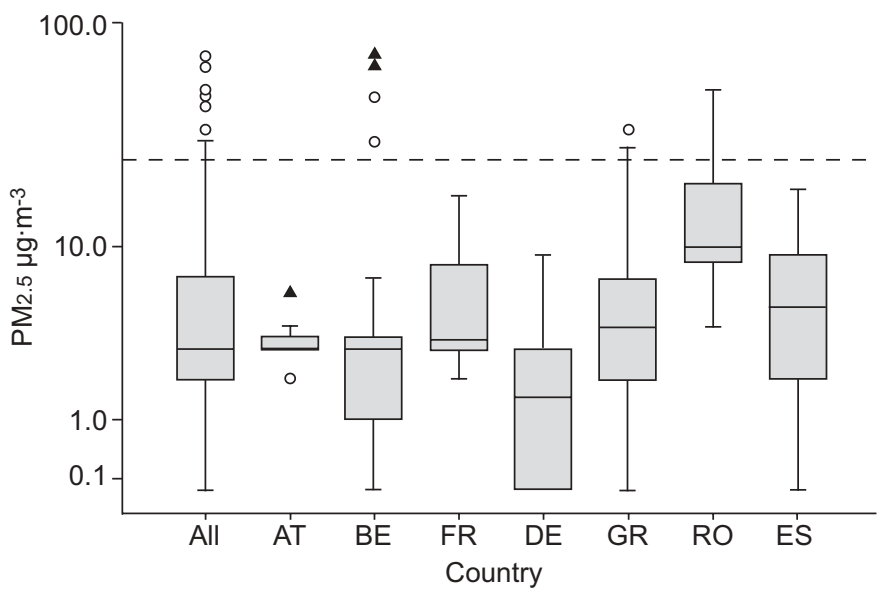

FIGURE 1. Boxplot showing distribution of particle with a $50 \%$ cut-off aerodynamic diameter of $2.5 \mu \mathrm{m}$ (PM2.5) concentrations (log scale) in 30 European hospitals by country, 2007. Boxes represent median and interquartile range; vertical bars represent values within 1.5 times the interquartile range $(O$ : outliers; $\mathbf{\Lambda}$ : extreme values; - - - -: 24-h mean limit recommended by the World Health Organization outdoor air quality guideline $\left.\left(25.0 \mu \mathrm{g} \cdot \mathrm{m}^{-3}\right)\right)$. All: all countries ( $n=199)$; AT: Austria $(n=12) ;$ BE: Belgium $(n=36) ;$ FR: France $(n=8) ;$ DE: Germany $(n=30)$; GR: Greece $(n=59) ; R O:$ Romania $(n=24)$; ES: Spain $(n=30)$.

Although all of the hospitals in the present study had implemented tobacco control policies following the ENSH code and standards, they did not have the same level of restriction, enforcement and fulfilment due to inter-country differences in legislation [3]. For example, smoking rooms inside hospitals were permitted in Austria, Belgium, Germany and Greece (table 2). Differences in baseline tobacco consumption among the population and the anti-smoking climate should also be taken into account. For example, Greece and Austria had high smoking prevalences, and, with Germany, had the lowest scores on the Tobacco Control Scale (table 1) [13]. These facts could well explain the different levels of SHS found in some areas in some hospitals.

Most of the measurements were below the 24-h mean limit recommended by the WHO and US Environmental Protection Agency for both outdoor and indoor air [15, 16]. The chemical composition of outdoor pollutants can differ from that of the indoor air measured in the hospitals. Outdoor PM2.5 concentrations used to be higher than indoor levels, although the time of exposure should also be considered for risk assessment. Moreover, the air quality guidelines refer to $24-\mathrm{h}$ or annual mean level, instead of the present spot measures. Although the site of exposure, indoors or outdoors, determines the composition of the air and concentration of the various pollutants, it does not directly affect the exposure-response relationship [15, 17].

Few studies have assessed SHS in hospitals. A pioneering study that measured airborne nicotine concentrations in 22 hospitals in seven European cities (Vienna (Austria), Paris (France), Athens (Greece), Florence (Italy), Porto (Portugal), Barcelona (Spain) and Örebro (Sweden)) during 2001-2002 showed low but detectable SHS exposure in hospitals $[5,18,19]$. Similar surveys conducted in 11 Latin American countries and China, including one hospital in each country between 2002 and 2006, also showed low but quantifiable nicotine concentrations

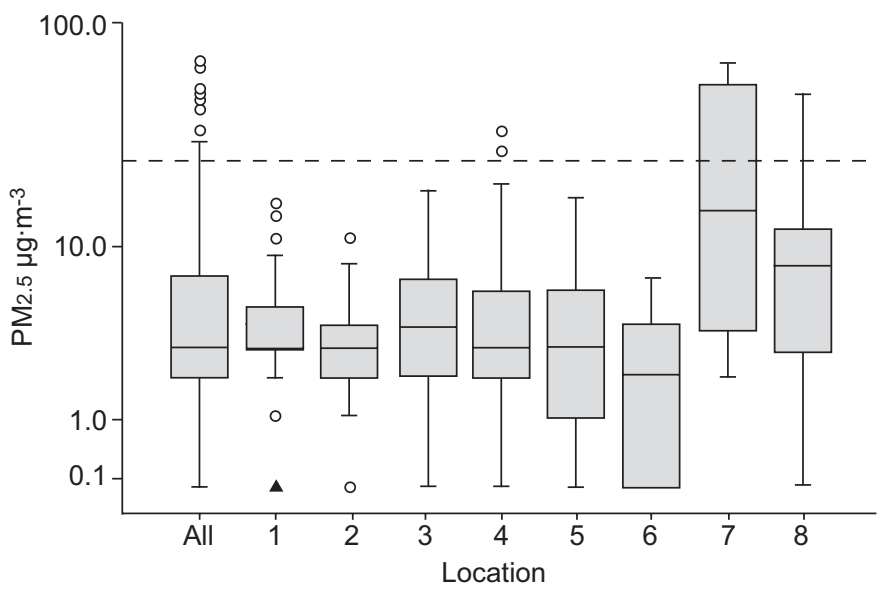

FIGURE 2. Boxplot showing distribution of particle with a $50 \%$ cut-off aerodynamic diameter of $2.5 \mu \mathrm{m}$ (PM2.5) concentrations (log scale) in 30 European hospitals by location of measurement, 2007. Boxes represent median and interquartile range; vertical bars represent values within 1.5 times the interquartile range $(\mathrm{O}$ : outliers; $\boldsymbol{\Delta}$ : extreme values; - - - -: 24-h mean limit recommended by the World Health Organization outdoor air quality guideline $\left.\left(25.0 \mu \mathrm{g} \cdot \mathrm{m}^{-3}\right)\right)$. All: all places $(\mathrm{n}=199) ; 1$ : hall (main entrance) $(\mathrm{n}=30) ; 2$ : emergency department waiting room ( $n=29)$; 3 : internal medicine hospitalisation unit $(n=22)$; 4: cafeteria $(n=27) ; 5$ : fire escape $(n=22) ; 6$ : general surgery hospitalisation unit $(n=22) ; 7$ : smoking area $(n=8) ; 8$ : other places $(n=39)$.

[20-23]. In a previous study in Catalonia, Spain, low levels of airborne nicotine were found in 44 public hospitals before the new Spanish tobacco control law came into force in 2006, which subsequently mostly decreased to unquantifiable concentrations after the ban [6]. However, PM2.5 concentrations have been scantily used in the monitoring of SHS in hospitals, except for some pilot experiences in Italy [24] and Greece [25]. These studies indicate that measurement of PM2.5 concentrations is a feasible and sensible method of SHS assessment in hospitals.

Some limitations of the present study merit consideration. First, the sample of participating hospitals was small (even considering that this is the first study to systematically survey 30 hospitals in different countries), and hospitals were recruited using a convenience framing approach and not selected at random. An attempt was made to ensure internal validity of the measurements by selecting the participating hospitals, given the complexity of the multi-country study. Secondly, a standard and accepted methodology was used to measure PM2.5 levels, by means of a commercial particle size monitor. The same monitor was used in all of the hospitals, and the local researchers in charge of the measurements were trained using a common protocol. Climatic conditions may have changed from hospital to hospital and country to country given that the field work was extended over several months. However, the mean temperature during measurements in all of the countries was $22.1^{\circ} \mathrm{C}$, and the mean relative humidity was $39.6 \%$, without huge variations across countries. Although 2min measurements were performed in each location, 10-20min mean measurements have been used in other studies. However, the reliability of the recordings was warranted by the good consistency of the different data from smoke-free locations of the same hospital on the same day, such as measurements in halls, emergency department waiting rooms 
and internal medicine hospitalisation units. The differences in PM2.5 concentrations found between locations where smoking was forbidden and those where it was permitted are also an indicator of the reliability of measurements. PM2.5 variations in hospitals are supposed to be very small in comparison to measurements carried out in other more polluted environments, such as pubs or bars, where mean concentrations over long periods of time are preferred. Thirdly, the number of sampling locations within each hospital was limited to six common places. It was not possible to survey more locations for operational reasons. However, this distribution of samples provided a good estimate of SHS levels in a previous study [5], and prevents an excessive variety of locations, which would make comparisons by location across countries unfeasible. Finally, there was a failure to obtain outdoor measurements for comparison with in-hospital measurements, although, given the low levels obtained indoors, the comparison group would have been almost useless. Last but not least, in the interpretation of the results, it should be taken into account that SHS is not the only source of indoor particulate matter, although it is considered its main contributor.

In conclusion, exposure to SHS, as measured by mean PM2.5 level, is very low across the present sample of European hospitals. Use of PM2.5 concentration as a marker of exposure to SHS appears to be a feasible method of comparing compliance with smoke-free regulations in hospitals both within and between countries. Periodical surveys of SHS exposure in hospitals following a common, standard and easy to implement protocol should be developed and promoted by the European public health authorities.

\section{SUPPORT STATEMENT}

The European Network for Smoke-free Hospitals received funds from the European Commission (contract No. 2005329). E. Fernández, M. Fu, J.M. Martínez-Sánchez, and C. Martínez are funded by the Ministry of Health (Red Temática de Investigación en Cáncer; RD06/0020/ 0089), Government of Spain, and Ministries of Science and Universities (SGR200500646) and Health (account 20008) of the Government of Catalonia. M.J. López and M. Nebot are funded by the Instituto de Salud Carlos III (Centro de Investigación Biomédica en Red en Epidemiología y Salud Pública; CB06/02/0032). B. Dautzenberg is supported by a grant from the French government.All of the authors participated in study design and writing of the protocol. C. Martínez and A. Ouranou coordinated data collection in the participating hospitals. M. Fu, M.J. López and M. Nebot supervised and performed quality control procedures. M. Fu, J.M. Martínez-Sánchez, M.J. López and C. Martínez administered and prepared the database. E. Fernández and J.M. Martínez-Sánchez analysed the data. All of the coauthors contributed to the interpretation of results. E. Fernández drafted the manuscript, which was critically revised by all of the coauthors. All co-authors approved the final version of the manuscript. E. Fernández is the guarantor.

\section{STATEMENT OF INTEREST}

A statement of interest for C. Martínez can be found at www.erj. ersjournals.com $/ \mathrm{misc} /$ statements.dtl

\section{ACKNOWLEDGEMENTS}

The authors would like to acknowledge the role of the local coordinators in facilitating data collection.

Participating hospitals. Austria: Krankenhaus Hietzing mit Neurologischem Zentrum Rosenhügel, Vienna. Belgium: AZ Sint
Lucas, Brugge; CHU Saint-Pierre, Brussels; Institut Jules Bordet, Brussels; Hôpital Erasme, Brussels; and AZ Sint Blasius, Dendermamdem. France: Hôpital Cochin, Paris; Hôtel-Dieu de Paris, Paris; and Centre Hospitalier Universitaire de Caen, Caen. Germany: Kreiskrankenhaus Osterholz, Osterholz; Evangelisches Krankenhaus Köln-Weyertal, Köln; Klinikum Dorothea Christiane Erxleben Quedlinburg, Quedlinburg; Kreiskliniken Aschersleben, Aschersleben; and Krankenhaus Sangerhausen, Sangerhausen. Greece: Tzaneio General Hospital, Pireaus; Evangelismos General Hospital, Agia Olga/Konstadopouleio, Geniko Kratiko/Genimatas, IKA Athinon, Agia Sofia Children's Hospital, and Laiko General Hospital, all Athens. Romania: Bucur Maternity Hospital, Universitary Maternity Hospital, Maternity Hospital, and Marius Nasta National Institute of Pneumology, all Bucharest. Spain: Institut Català d'Oncologia, L'Hospital et de L lobregat; Hospital de Mollet, Mollet del Vallès; Hospital de Badalona, Barcelona; Hospital Clínic i Provincial de Barcelona, Barcelona; and Hospital Sant Joan de Déu Esplugues de Wobregat.

\section{REFERENCES}

1 World Health Organization, International Agency for Research on Cancer. IARC Monographs on the Evaluation of Carcinogenic Risks to Humans. Vol. 83. Tobacco smoke and involuntary smoking. Lyon, International Agency for Research on Cancer 2004.

2 US Department of Health and Human Services. The Health Consequences of Involuntary Exposure to Tobacco Smoke: a Report of the Surgeon General. Atlanta, US Department of Health and Human Services, Centers for Disease Control and Prevention, Coordinating Center for Health Promotion, National Center for Chronic Disease Prevention and Health Promotion, Office on Smoking and Health, 2006.

3 World Health Organization Regional Office for Europe. The European tobacco control report 2007. Copenhagen, World Health Organization, 2007.

4 Garcia M, Méndez, Martínez C, et al. Implementing and complying with the Smoke-free Hospitals Project in Catalonia, Spain. Eur J Cancer Prev 2006; 15: 446-452.

5 Nebot M, Lopez MJ, Gorini G, et al. Environmental tobacco smoke exposure in public places of European cities. Tob Control 2005; 14: 60-63.

6 Fernandez E, Fu M, Martínez C, et al. Secondhand smoke in hospitals of Catalonia (Spain) before and after a comprehensive ban on smoking at the national level. Prev Med 2008; 7: 624-628.

7 Benowitz NL. Biomarkers of environmental tobacco smoke exposure. Environ Health Perspect 1999; 107: Suppl. 2, 349-355.

8 Gorini G, Gasparrini A, Fondelli M, et al., Second-hand Smoke (SHS) Markers: Review of Methods for Monitoring Exposure Levels. Brussels, European Network for Smoking Prevention, 2005.

9 Repace JL, Hyde JN, Brugge D. Air pollution in Boston bars before and after a smoking ban. BMC Public Health 2006; 6: 266.

10 Semple S, Creely KS, Naji A, et al. Secondhand smoke levels in Scottish pubs: the effect of smoke-free legislation. Tob Control 2007; 16: $127-132$.

11 Hyland A, Travers MJ, Dresler C, et al. A 32-country comparison of tobacco smoke derived particle levels in indoor public places. Tob Control 2008; 17: 159-165.

12 Hyland A, Travers MJ, Repace JL. 7 city air monitoring Study, March-April 2004. Buffalo, Roswell Park Cancer Institute, 2004.

13 Progress in Tobacco Control in 30 European Countries, 2005 to 2007. Berne, Swiss Cancer League, Association of European Cancer Leagues, European Network for Smoking Prevention, 2007. 
14 Boffi R, Ruprecht A, Mazza R, et al. A day at the European Respiratory Society Congress: passive smoking influences both outdoor and indoor air quality. Eur Respir J 2006; 27: 862-863.

15 World Health Organization Regional Office for Europe. Air Quality Guidelines for Europe. 2nd Edn. Copenhagen, World Health Organization, 2000.

16 US Environmental Protection Agency. PM Standards Revision 2006. Washington, DC, US Environmental Protection Agency, 2006.

17 World Health Organization Regional Office for Europe. Air Quality Guidelines for Europe. Update. Copenhagen, World Health Organization, 2005.

18 Lopez MJ, Nebot M, Salles J, et al. Medición de la exposición al humo ambiental de tabaco en centros de enseñanza, centros sanitarios, medios de transporte y lugares de ocio. [Measurement of exposure to environmental tobacco smoke in education centres, health centres, transport facilities and leisure places.]. Gac Sanit 2004; 18: 451-457.

19 Gorini G, Fondelli MC, Lopez MJ, et al. Esposizione a fumo passivo in alcuni luoghi pubblici in Firenze. [Environmental tobacco smoke exposure in public places in Florence, Italy.] Epidemiol Prev 2004; 28: 94-99.
20 Navas-Acien A, Peruga A, Breysse P, et al. Secondhand tobacco smoke in public places in Latin America, 2002-2003. JAMA 2004; 291: 2741-2745.

21 Barnoya J, Mendoza-Montano C, Navas-Acien A. Secondhand smoke exposure in public places in Guatemala: comparison with other Latin American countries. Cancer Epidemiol Biomarkers Prev 2007; 16: 2730-2735.

22 Barrientos-Gutierrez T, Valdes-Salgado R, Reynales-Shigematsu LM, et al. Exposición involuntaria al humo de tabaco en lugares públicos de la Ciudad de México. [Involuntary exposure to tobacco smoke in public places in Mexico City.] Salud Publica Mex 2007; 49: Suppl. 2, S205-S212.

23 Stillman F, Navas-Acien A, Ma J, et al. Second-hand tobacco smoke in public places in urban and rural China. Tob Control 2007; 16: 229-234.

24 Nardini S, Cagnin R, Invernizzi G, et al. Indoor particulate matter measurement as a tool in the process of the implementation of smoke-free hospitals. Monaldi Arch Chest Dis 2004; 61: 183-192.

25 Vardavas C, Mpouloukaki I, Linardakis M, et al. Second hand smoke exposure and excess heart disease and lung cancer mortality among hospital staff in Crete, Greece: a case study. Int J Environ Res Public Health 2008; 5: 125-129. 